\title{
Fabry disease: a morphologic study of 11 cases
}

\author{
Edgar G Fischer, Michael J Moore and Donna J Lager \\ Department of Laboratory Medicine and Pathology, Mayo Foundation, Rochester, MN, USA
}

\begin{abstract}
Fabry disease is a metabolic disorder caused by the genetic deficiency of alpha-galactosidase A. Deposition of glycosphingolipids in podocytes, endothelial cells, and other cell types leads to formation of myelin-like inclusions, which are the hallmark of the disease. In most untreated males, the disorder progresses to endstage kidney disease. Fabry disease is rare, and no renal biopsy series focusing on pathologic findings has been published in the past 25 years. We retrieved kidney biopsies diagnosed with Fabry disease from our files, and reviewed clinical data as well as the light and electron microscopy. In total, 11 patients were identified: six male subjects aged 17-43 years and five female subjects aged 30-73 years. On average, male patients presented more than 10 years earlier then female patients. A total of 10 patients had proteinuria, two with the nephrotic syndrome. Four male and three female patients had decreased renal function. Light microscopy showed vacuolization of the podocyte cytoplasm and variable glomerular sclerosis. Older patients and males had more advanced glomerular and interstitial sclerosis, but three of the five female patients also had advanced renal disease. Electron microscopy showed the characteristic myelin-like inclusions most prominently in the podocyte cytoplasm. Seven patients also had podocyte foot process effacement. A second type of deposit, unexpected and conspicuous, was identified in three males, and found to be associated with glomerular basement membrane duplications. These deposits were composed of layered membrane-like material, and therefore morphologically distinct from myelin-like inclusions. They probably represent remnants of damaged endothelial cells.
\end{abstract}

Modern Pathology (2006) 19, 1295-1301. doi:10.1038/modpathol.3800634; published online 23 June 2006

Keywords: Fabry disease; alpha-galactosidase A; glycosphingolipidosis; kidney biopsy; myelin-like inclusions

Fabry disease is a rare metabolic disorder caused by the genetic deficiency of the lysosomal hydrolase alpha-galactosidase A. Intracellular accumulation of inclusion bodies with a myelin-like appearance is the pathologic hallmark of the disease. ${ }^{1-3}$ The estimated incidence ranges from 1:40000 to 1:60 000 males. ${ }^{4}$ The alpha-galactosidase A gene is located on the long arm of chromosome X. Several different point mutations or gene rearrangements have been identified as the cause of the disease. ${ }^{1,3}$ Because of its X-linked inheritance, the disease is completely expressed in hemizygous males. The enzyme defect causes progressive accumulation of glycosphingolipids in the vascular endothelium and other cell types, which leads to renal, cardiac, and cerebral dysfunction and early death. ${ }^{4}$ In untreated hemizygous males, severe deterioration of renal

Correspondence: Dr EG Fischer, MD, PhD, Department of Pathology, MSC08 4640, University of New Mexico, Albuquerque, NM 87131, USA.

E-mail: efischer@salud.unm.edu

Received 14 December 2005; revised and accepted 25 April 2006; published online 23 June 2006 function usually occurs in the second and third decades of life, with progression to end-stage renal disease. $^{1,3}$ A 'renal variant' phenotype was recently described in male patients who developed end-stage renal disease without the classic extra-renal symptoms. ${ }^{5}$ In recent years, enzyme replacement therapy has become available, and its clinical safety and efficacy has been reported in clinical trials. ${ }^{3,4,6-8}$ Renal biopsy findings were reported in the context of several treatment studies, in which patients were selected for preserved or mildly abnormal renal function. . $^{3,4,6,7}$

Fabry ${ }^{9}$ and Anderson ${ }^{10}$ first described the disease in 1898 as angiokeratoma corporis diffusum universale. Its pathobiochemistry is well established today. ${ }^{1-3}$ In normal cells, the enzyme alpha-galactosidase A is located in lysosomes. It is involved in the catabolism of globotriaosylceramide and related glycosphingolipids by removing alpha-linked galactosyl residues from ceramide trihydroxide. The enzyme deficiency manifests as a glycosphingolipidosis with progressive accumulation of glycosphingolipids and deposition of inclusion bodies with a myelin-like appearance in lysosomes. ${ }^{1,2,11}$ The myelin-like inclusions have a characteristic 
ultrastructural appearance with concentric osmiophilic lamellations. In the kidney, these inclusions are most prominently deposited in podocytes, but are also found in mesangial cells, glomerular endothelial cells, tubular epithelial cells, and in interstitial blood vessels. ${ }^{1-3,11}$ Inclusion deposits in podocytes cause cellular injury, which is followed by capillary wall thickening, progressive mesangial matrix expansion, glomerulosclerosis, and end-stage renal disease. ${ }^{1-3,11}$

The pathologic features of Fabry disease have been studied by light microscopy, histochemistry, ${ }^{12}$ and electron microscopy. Pathologic changes were described in heterozygous female subjects ${ }^{13}$ and in a renal allograft. ${ }^{14}$ However, most pathologic studies were conducted on autopsy or pretransplantation nephrectomy specimens. So far, only one series studying the pathology of renal biopsies has been published, which was more than 25 years ago. ${ }^{15}$ Here, we report pathologic findings in a series of renal biopsies from 11 patients with Fabry disease.

\section{Materials and methods}

Kidney biopsies from patients with Fabry disease were retrieved from the Mayo Clinic files between 1997 and 2003. Clinical data and pathologic findings at routine light microscopy, electron microscopy, and immunofluorescent microscopy were reviewed. Routine light microscopic examination was performed on hematoxylin and eosin, periodic acid-Schiff, Jones methenamine silver, and trichrome stains. Histologic findings were scored by light microscopy with respect to glomerular and interstitial changes as: $0+$, no significant change; $1+$, mild; $2+$, moderate; and $3+$, severe change. We also reviewed the plastic-embedded toluidine bluestained sections that were prepared as survey sections for electron microscopy. Routinely performed electron microscopic images were reviewed in all cases. None of our patients had a history of taking medications known to cause Fabry-like morphologic changes, such as chloroquine, amiodarone, or aminoglycosides.

\section{Results}

We identified 11 cases of Fabry disease in our renal biopsy files, and their clinical and laboratory data are summarized in Table 1 . Six patients were male, ranging in age from 17 to 43 years (mean 36.3, median 39.5), and five patients were female and between 30 and 73 years of age (mean 49.8, median $54)$. Two males in this series were brothers (cases 4 and 5). Measurements of 24-h urinary protein were available in 10 patients and all had significant proteinuria, two with documented nephrotic syndrome. Eight patients had declining renal function detected by creatinine clearance measurement or increased serum creatinine levels. Three patients were hypertensive, one of which developed malignant hypertension (Table 1).

At routine light microscopy, swollen podocytes with vacuolated cytoplasm were a consistent finding (Figure 1a). These vacuoles are left behind after the glycosphingolipid deposits are extracted by the tissue processing reagents. Mesangial hypercellularity was present in all male and two female patients, which was usually mild, and severe only in the case with concomitant malignant hypertension (Table 2). Almost all patients had increased mesangial matrix with segmental glomerular sclerosis and obsolescent glomeruli on their biopsies (Table 2). These changes were more pronounced in male and older patients than in female and younger patients. However, three of our female patients, ages 54 years and older, had advanced disease at presentation (cases 8, 9, and 11) (Table 2). Synechiae between segmental tuft lesions and Bowman's capsule were present in three patients (cases 1, 7, and 9). One 17-year-old male presented with early disease and had only mesangial widening, but no glomerular or interstitial

Table 1 Clinical and laboratory data of 11 patients with Fabry disease

\begin{tabular}{|c|c|c|c|c|c|c|c|}
\hline Case & Sex & Age & $\begin{array}{l}\text { Serum creatinine } \\
(\mathrm{mg} / \mathrm{dl})\end{array}$ & $\begin{array}{c}\text { Creatinine } \\
\text { clearance }(\mathrm{ml} / \mathrm{min})\end{array}$ & $\begin{array}{l}\text { Urine protein } \\
\quad(\mathrm{g} / 24 \mathrm{~h})\end{array}$ & Hypertension & Others \\
\hline 1 & $\mathrm{M}$ & 42 & 5.1 & 14 & 4.3 & & \\
\hline 2 & $\mathrm{M}$ & 39 & 1 & 86 & $2-4$ & Yes & $\begin{array}{l}\text { Nephrotic syndrome, cardiomyopathy, } \\
\text { skin rash }\end{array}$ \\
\hline 3 & $\mathrm{M}$ & 43 & 1.1 & & 2.65 & No & $2-3+$ edema from knee down \\
\hline 4 & M & 37 & 4 & & & & Has brother with Fabry disease (case 5) \\
\hline 5 & $\mathrm{M}$ & 40 & 3.4 & & $3-4$ & Sudden, malignant & Has brother with Fabry disease (case 4) \\
\hline 6 & M & 17 & & & $\begin{array}{l}\text { Nephrotic } \\
\text { range }\end{array}$ & & \\
\hline 7 & $\mathrm{~F}$ & 30 & & & $2-3$ & Yes & \\
\hline 8 & $\mathrm{~F}$ & 73 & 1.9 & 44 & 3.6 & Mild & $\begin{array}{l}\text { Nephrotic syndrome, type II diabetes } \\
\text { with neuropathy }\end{array}$ \\
\hline 9 & $\mathrm{~F}$ & 54 & 2.2 & 37 & 2 & & \\
\hline 10 & $\mathrm{~F}$ & 37 & 1 & Declining & 1.7 & & \\
\hline 11 & $\mathrm{~F}$ & 55 & 6.4 & 15 & 3.76 & & \\
\hline
\end{tabular}



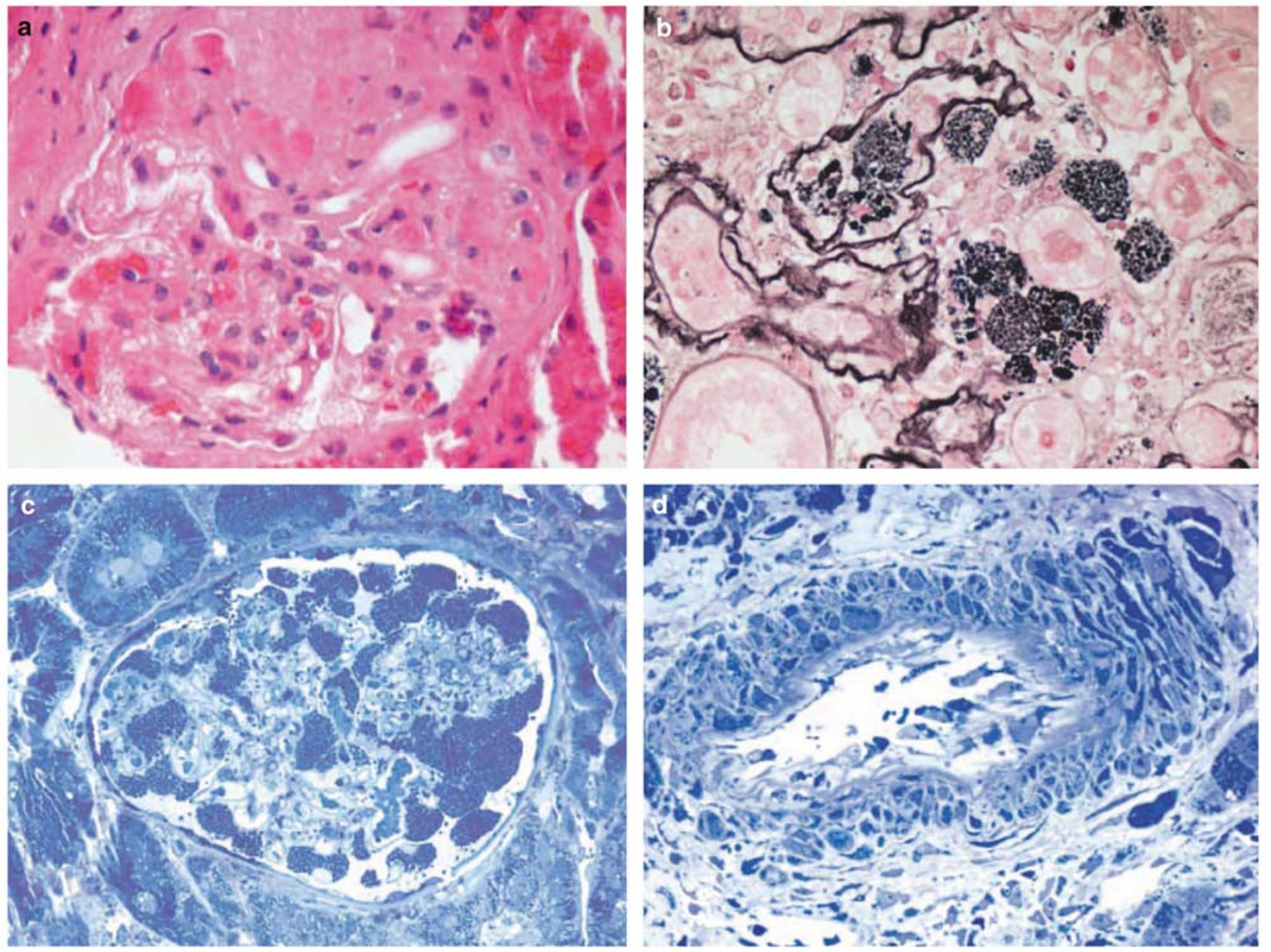

Figure 1 Light microscopic changes in Fabry disease. (a) Glomerulus with foamy podocyte cytoplasm and segmental sclerosis (H\&E stain, $\times 600)$. (b) Silver positive inclusions in tubular epithelial cells and interstitial macrophages (Jones methenamine silver stain, $\times 600$ ). (c) Glomerulus with abundant podocyte inclusions (plastic-embedded section, Toluidine blue stain, $\times 600$ ). $($ d) Cytoplasmic inclusions in smooth muscle and endothelial cells of an interlobular-type artery (plastic-embedded section, Toluidine blue stain, $\times 400$ ).

sclerosis (case 6). In the tubulo-interstitial compartment, nine patients had significant interstitial fibrosis, a chronic inflammatory infiltrate, and tubular atrophy (Table 2). Interstitial arteriolar fibrosis was of a similar extent (Table 2).

Two patients had abnormalities of a second disease process superimposed on the changes of Fabry disease. A 40-year-old male (case 5) had histologic changes of malignant hypertension with severe concentric intimal fibrosis and myxoid changes of interstitial blood vessels. Nonsclerotic glomeruli on his biopsy showed hypersegmentation, hyperlobulation, and severe glomerular hypercellularity with obliteration of capillary loops. A 73-yearold female (case 8) had concomitant type II diabetes mellitus. She had increased mesangial matrix with early nodule formation and peri-glomerular fibrosis.

The myelin-like inclusions stood out particularly well on toluidine blue-stained thin sections (Figure 1c and $\mathrm{d}$ ). The most prominent location of the inclusions was in podocytes (Figure 1c). Owing to the small number of cases in this series, we did not did not quantify the numbers of abnormal podocytes in individual cases. However, it appeared that myelin-like inclusions were more numerous in glomeruli from male compared to female patients. Inclusions in the parietal glomerular epithelium and tubular epithelium (Figure 1c) were evident in both male and female subjects. Involvement of tubular epithelium appeared more pronounced in the distal nephrons. Interstitial blood vessels showed myelinlike inclusions in endothelial cells and medial smooth muscle cells (Figure 1d), again more prominent in male than in female subjects. Immunofluorescent microscopy was negative in six cases and revealed weak nonspecific glomerular IgM and C3 staining in five cases (data not shown).

Ultrastructurally, podocytes demonstrated numerous lamellated myelin-like inclusions, which markedly distended their cytoplasm (Figure 2a and b). Myelin-like inclusions had a characteristic appearance with concentric, dark osmiophilic 
lamellations with an onion-skin-like appearance (Figure 2a and b). Ovoid inclusions with a more parallel arrangement of the dense layers, known as zebra bodies, were also present (Figure $2 b$ and $c$ ). Inclusions were surrounded by a single unit membrane, indicating their location in lysosomes (Figure 2c and d). Myelin-like inclusions were present in smaller numbers in mesangial cells, glomerular endothelial cells (Figure 2a and b), and parietal epithelial cells. Interestingly, three male patients also had a morphologically distinct type of deposits. These deposits were located on the subendothelial aspect of the glomerular basement membrane and associated with basement membrane duplications (cases 1, 2, and 4) (Figure 2c-e). They were composed of membrane-like material arranged in geographic layers, resembling contour lines on a map (Figure 2c-e). Podocytes showed foot process effacement with microvillous transformation in seven cases, and its presence correlated with the clinical presence of proteinuria or the nephrotic syndrome. Capillary loops were of normal thickness in four cases, wrinkled and focally thickened in four other cases, and collapsed with advanced sclerosis in three cases. The mesangial matrix was expanded in almost all cases. Myelin-like bodies were also present in tubular epithelial cells, endothelial cells, and vascular smooth muscle cells. The male patient with malignant hypertension (case 5) had subendothelial lucencies and obliteration of many capillary loops. The female patient with types II diabetes mellitus (case 8) had thickened and wrinkled capillary walls, luminal narrowing, and increased mesangial matrix.

\section{Discussion}

We reviewed pathologic findings in 11 patients who were first diagnosed with Fabry disease on renal biopsy. Eight patients had severely impaired renal function at diagnosis. A total of 10 patients had significant proteinuria, two with the nephrotic syndrome (Table 1). Hemizygous male subjects were on average more than 10 years younger than female subjects, and generally had more severe pathologic changes on biopsy (Table 2).

Routine light microscopy showed distention of podocytes by abundant cytoplasmic vacuoles. More advanced cases had increased mesangial matrix, segmental glomerulosclerosis with occasional synechiae, and globally sclerotic glomeruli (Figure 1a, Table 2), as described by others. ${ }^{1,2,11,15,16}$ The majority of cases (six male and two female subjects) demonstrated mild glomerular hypercellularity (Table 2). Three female subjects had advanced renal disease at presentation (Tables 1 and 2), although female subjects were generally thought to have a milder disease course. A recent examination of carrier female subjects has revealed more frequent disease manifestations than previously expected. ${ }^{17}$ 

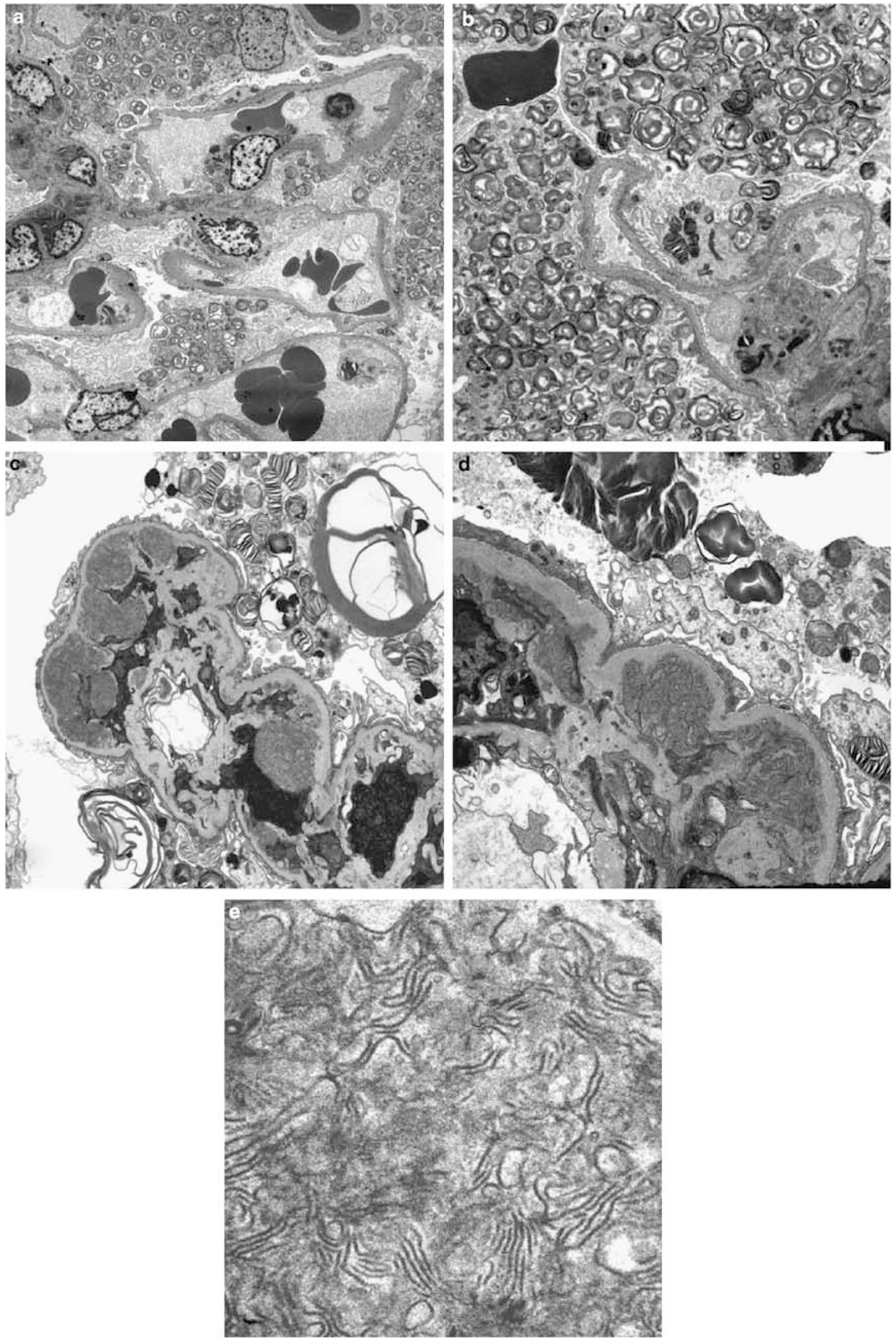

Figure 2 Electron microscopic changes in Fabry disease. (a, b) Myelin-like inclusions in podocyte cytoplasm $(\mathbf{a} \times 1800 ; \mathbf{b} \times 4200)$. (c-e) Subendothelial deposits composed of membrane-like material associated with glomerular basement membrane duplications $(\mathbf{c} \times 4200$; d $\times 7400$; $\times 24500$ ). 
The most prominent location of myelin-like inclusions was in podocytes, as illustrated on toluidine blue-stained sections (Figure 1c). Some authors have characterized the glomerular distribution of abnormal podocytes as homogeneous in male and as irregular in female subjects. The Lyon's hypothesis with random $\mathrm{X}$ chromosome inactivation in the female subject was discussed as a possible explanation. ${ }^{11,15}$ The tubulo-interstitium in more advanced cases showed abundant myelin-like deposits in tubular epithelial cells, vascular endothelial, and smooth muscle cells (Figure 1d), and in interstitial collections of macrophages (Figure 1b).

Electron microscopy demonstrated abundant lamellated myelin-like inclusions in podocytes as the hallmark of the disease (Figure 2a and b). Inclusions in mesangial cells and glomerular endothelial cells were a much more subtle finding. A single unit membrane surrounded the myelin-like bodies indicating their presence in lysosomes (Figure 2c and d). ${ }^{1,11,15}$ The myelin-like bodies were round and composed of concentric dense layers. We estimated their size at approximately $0.3-10 \mu \mathrm{m}$ in diameter. Zebra bodies were ovoid with dense layers in parallel arrays (Figure 2b and c). ${ }^{1,15}$ Several authors analyzed the dense concentric layers of myelin-like inclusions at very high magnification, and found them to be composed of alternating dark and clear laminations with a periodicity between 4 and $10 \mathrm{~nm}$ (40 and $100 \AA$ ). . $^{2,12,15}$

Podocyte foot process effacement and corresponding proteinuria was present in almost all our patients, which are features of more advanced disease. ${ }^{1,11,16}$ Accordingly, the authors of a biopsy series of patients with early renal changes in Fabry disease noted that their foot processes were normal and patients mostly lacked proteinuria. ${ }^{15}$ Three males in our series had an additional remarkable ultrastructural finding (cases 1, 3, and 4). Conspicuous deposits of membrane-like material were seen associated with glomerular basement membrane duplications. These deposits were morphologically distinct from the classical myelin-like inclusions. They were composed of membrane-like material arranged in geographic layers, reminiscent of the contour lines on a map, and with a granular texture between these layers (Figure 2c-e). A similar finding was discussed in one previous study (Figures 6 and 7 in Gubler et $a l^{15}$ ). Origin and significance of these membrane-like deposits are unclear, but their location suggests that they might be remnants of damaged endothelial cells. These membrane-like deposits were also described in the walls of interstitial arteries, and were thought to be remnants of damaged smooth muscle cells. ${ }^{15}$ Cohen and Adler $^{1}$ also described striated membranous structures permeating arterial walls, and felt that they represent remnants of necrotic smooth muscle cells. They did not describe such structures in glomeruli.

Fabry disease has been reported in association with other renal diseases, such as lupus erythe- matosus, ${ }^{18,19}$ IgA nephropathy, ${ }^{20,21}$ pauci-immune-,${ }^{22}$ and immune complex-mediated necrotizing crescentic glomerulonephritis. ${ }^{23}$ Recent reports described myelin-like deposits in the podocytes of two female subjects who took chloroquine for autoimmune disorders. ${ }^{24,25}$ Various other drugs including amiodarone are known to cause phospholipidoses that mimic Fabry disease. ${ }^{1,24}$ Therefore, myelin-like inclusions are not entirely specific for Fabry disease, although they are highly characteristic.

In summary, we report renal pathologic findings in a series of 11 patients with Fabry disease. Surprisingly, three of our five female subjects had advanced renal disease. We discuss the unusual ultrastructural finding of subendothelial membranelike deposits, a finding that is not well established in the literature on Fabry disease. At a time where there is much interest in enzyme replacement therapy, we hope that this discussion of the pathology in Fabry disease will contribute to our understanding of this rare metabolic disorder.

\section{References}

1 Cohen AH, Adler SG. Nail-patella syndrome (osteoonychodysplasia), lipodystrophy, Fabry's disease (angiokeratoma corporis diffusum universale), and familial lecithin-cholesterol acyltransferase deficiency. In: Tisher CC, Brenner BM (eds). Renal Pathology, 2nd edn. Lippincott Company: Philadelphia, 1994, pp 1267-1289.

2 Bernstein J, Churg J. Heritable metabolic diseases. In: Jennette JC, Olson JL, Schwartz MM, Silva FG (eds). Heptinstall's Pathology of the Kidney, 5th edn. Lippincott-Raven Publishers: Philadelphia, 1998, pp 1287-1320.

3 Branton MH, Schiffmann R, Sabnis SG, et al. Natural history of Fabry renal disease: influence of alphagalactosidase A activity and genetic mutations on clinical course. Medicine 2002;81:122-138.

4 Desnick RJ, Brady R, Barranger J, et al. Fabry disease, an under-recognized multisystemic disorder: expert recommendations for diagnosis, management, and enzyme replacement therapy. Ann Intern Med 2003; 138:338-346.

5 Nakao S, Kodama C, Takenaka T, et al. Fabry disease: detection of undiagnosed hemodialysis patients and identification of a 'renal variant'. Kidney Int 2003;64: 801-807.

6 Schiffmann R, Kopp JB, Austin III HA, et al. Enzyme replacement therapy in Fabry disease: a randomized controlled trial. JAMA 2001;285:2743-2749.

7 Eng CM, Guffon N, Wilcox WR, et al. Safety and efficacy of recombinant human alpha-galactosidase A-replacement therapy in Fabry's disease. N Engl J Med 2001;345:9-16.

8 Desnick RJ, Banikazemi M, Wasserstein M. Enzyme replacement therapy for Fabry disease, an inherited nephropathy. Clin Nephrol 2002;57:1-8.

9 Fabry J. Ein Beitrag zur Kenntnis der Purpura haemorrhagica nodularis (Purpura haemorrhagica Hebrae). Arch Dermatol Syphilol 1898;43:187. 
10 Anderson W. A case of angiokeratoma. Br J Dermatol 1898;10:113.

11 Sessa A, Toson A, Nebuloni M, et al. Renal ultrastructural findings in Anderson-Fabry disease. J Nephrol 2002;15:109-112.

12 Faraggiana T, Churg J, Grishman E, et al. Light- and electron-microscopic histochemistry of Fabry's disease. Am J Pathol 1981;103:247-262.

13 Farge D, Nadler S, Wolfe LS, et al. Diagnostic value of kidney biopsy in heterozygous Fabry's disease. Arch Pathol Lab Med 1985;109:85-88.

14 Popli S, Molnar ZV, Leehey DJ, et al. Involvement of renal allograft by Fabry's disease. Am J Nephrol 1987; 7:316-318.

15 Gubler MC, Lenoir G, Grunfeld JP, et al. Early renal changes in hemizygous and heterozygous patients with Fabry's disease. Kidney Int 1978;13: 223-235.

16 Alroy J, Sabnis S, Kopp JB. Renal pathology in Fabry disease. J Am Soc Nephrol 2002;13(Suppl 2): S134-S138.

17 MacDermot KD, Holmes A, Miners AH. AndersonFabry disease: clinical manifestations and impact of disease in a cohort of 60 obligate carrier females. J Med Genet 2001;38:769-775.
18 Rosenmann E, Kobrin I, Cohen T. Kidney involvement in systemic lupus erythematosus and Fabry's disease. Nephron 1983;34:180-184.

19 Rahman P, Gladman DD, Wither J, et al. Coexistence of Fabry's disease and systemic lupus erythematosus. Clin Exp Rheumatol 1998;16:475-478.

20 Yoshida A, Morozumi K, Takeda A, et al. Fabry-like laminated myelin body associated with IgA nephropathy. Nippon Jinzo Gakkai Shi 1994;36:1303-1307.

21 Kawamura O, Sakuraba H, Itoh K, et al. Subclinical Fabry's disease occurring in the context of IgA nephropathy. Clin Nephrol 1997;47:71-75.

22 Singh HK, Nickeleit V, Kriegsmann J, et al. Coexistence of Fabry's disease and necrotizing and crescentic glomerulonephritis. Clin Nephrol 2001;55:73-79.

23 Shimazu K, Tomiyoshi Y, Aoki S, et al. Crescentic glomerulonephritis in a patient with heterozygous Fabry's disease. Nephron 2002;92:456-458.

24 Muller-Hocker J, Schmid H, Weiss M, et al. Chloroquine-induced phospholipidosis of the kidney mimicking Fabry's disease: case report and review qof the literature. Hum Pathol 2003;34:285-289.

25 Albay D, Adler SG, Philipose J, et al. Chloroquineinduced lipidosis mimicking Fabry disease. Mod Pathol 2005;18:733-738. 